\title{
Set-membership state estimation with fleeting data
}

\author{
F. Le Bars, J. Sliwka, O. Reynet and L. Jaulin \\ ENSTA Bretagne, OSM, Lab-STICC, 2 rue François Verny, 29806 Brest.
}

\begin{abstract}
This paper deals with offline nonlinear state estimation where measurements are available only when some given equality conditions are satisfied. For this type of problems, which are often met in robot localization when sonar or radar are involved, the data are qualified as fleeting because the measurements are available only at some given unknown dates. In this paper, the first approach able to deal with nonlinear estimation with fleeting data is presented. An illustration related to offline robot localization with a laser rangefinder will be given.
\end{abstract}

Index Terms - Fleeting data, interval analysis, localization, observer, set-membership estimation.

\section{INTRODUCTION}

This paper deals with offline nonlinear state estimation in a bounded-error context (see e.g., [1], [5], [11], [17], [8], [22]), but here, we shall consider fleeting data, i.e., fugitive data that can appear at some given dates. More formally, a state estimation problem with fleeting data can be represented by a classical state equation with some visibility conditions:

$$
\left\{\begin{array}{l}
\dot{\mathbf{x}}(t)=\mathbf{f}(\mathbf{x}(t), t)+\mathbf{b}(t) \\
h(\mathbf{x}(t))=0 \Rightarrow g(\mathbf{x}(t)) \in \mathbb{W}(t)
\end{array}\right.
$$

where $t \in \mathbb{R}$ is the time, $\mathbf{x}(t)$ is the state vector, $\mathbf{b}(t)$ is the state noise vector which is assumed to belong to a known box $[\mathbf{b}], \mathbf{f}: \mathbb{R}^{n} \times \mathbb{R} \rightarrow \mathbb{R}^{n}$ is the evolution function, $h: \mathbb{R}^{n} \rightarrow \mathbb{R}$ is the visibility function and $g:$ $\mathbb{R}^{n} \rightarrow \mathbb{R}$ is the observation function. The function $\mathbb{W}(t)$, which is called a waterfall (see, e.g. [7]), is composed of measured compact subsets of $\mathbb{R}$ and encloses significant data only when some equality conditions are satisfied. As an example, we can consider the situation of a robot equipped with a laser rangefinder and a single punctual landmark $\mathbf{m}$. The rangefinder measures the distance $d$ to $\mathbf{m}$, only if the laser points exactly towards $\mathbf{m}$. The condition "points exactly" corresponds to an equality. If this equality is not satisfied, the rangefinder provides a data which is not significant for localization. We shall assume that the functions $\mathbf{f}, h, g$ are continuous and differentiable. A fleeting data point is a pair $(t, g(\mathbf{x}(t)))$ such that $h(\mathbf{x}(t))=0$. Figure 1 illustrates these notions. The four corresponding fleeting dates are $t_{1}, t_{2}, t_{3}, t_{4}$.

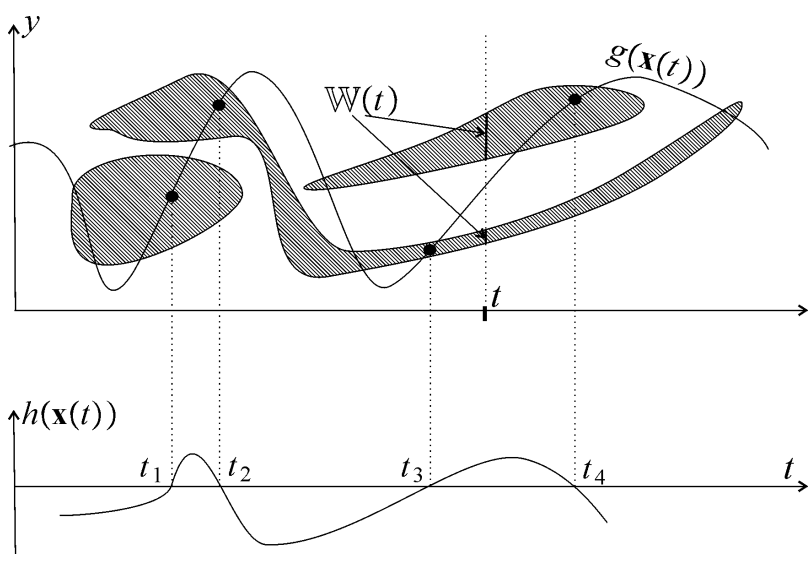

Fig. 1. The four fleeting data points $(t, g(\mathbf{x}(t)))$, represented by the four black dots are known to belong to the waterfall $\mathbb{W}(t)$. At time $t$, a fleeting data point is met only if the visibility condition $h(\mathbf{x}(t))=0$ is satisfied.

The existence and the location of the fleeting data points are unknown, but can be estimated through the waterfall $\mathbb{W}(t)$ and the state equations. Most of the elements of $\mathbb{W}(t)$ cannot be considered as significant. In practice, the waterfall is obtained from sensors and can be represented by an image (a lateral sonar image for instance). In the waterfall of Figure 2 the two black vertical segments indicate the part of the signal collected by a lateral sonar at times $t_{1}$ and $t_{2}$. At time $t_{1}$ the mark is detected (it is inside the circle) and at time $t_{2}$ the collected data are not related to the mark. Another way to understand the information provided by the image is that the mark cannot be inside the intersection between the black vertical segment and the grey area. As a result, with a waterfall, we do not detect the mark, we get zones where the mark cannot be.

The state estimation problem to be considered here is to find an envelope which encloses all state trajectories that are consistent with Equations (1), the waterfall $\mathbb{W}(t)$ and a feasible box for the initial condition. Even if state estimation with fleeting data can be considered as fundamental in robot localization, to our knowledge, it has never been studied before.

To solve our state estimation problem, we present in Section II a new contractor-based approach. Contrary to existing propagation methods where the domains are 


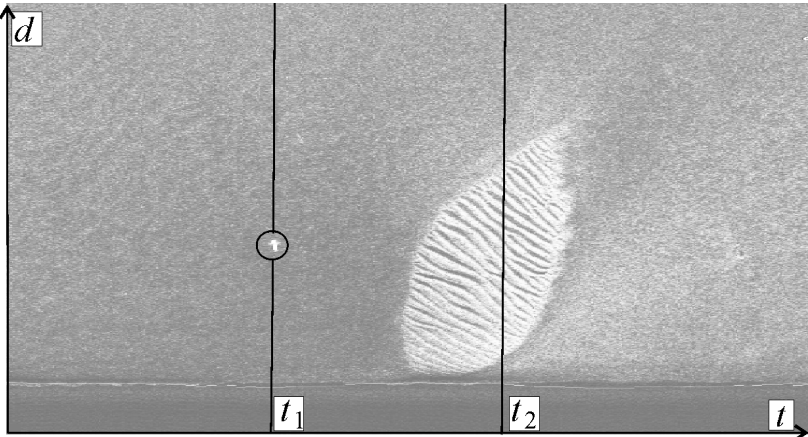

Fig. 2. A part of the waterfall collected by the portside lateral sonar of the underwater robot Redermor built by GESMA (Groupe Etude Sous-Marine de l'Atlantique)

either discrete, intervals or boxes, the domains to be considered are tubes which enclose trajectories. Section III introduces some theoretical tools to build contractors associated with the visibility condition. The resolution algorithm is presented on Section IV. Section V provides an illustrative example and Section VI concludes the paper.

\section{CONSTRAINT PROPAGATION With TUBES}

Many problems of estimation, control, robotics, and related fields can be represented by continuous constraint satisfaction problems (CSP) [12], [18], [23]. A CSP is composed of a set of variables $\mathcal{V}=\left\{x_{1}, \ldots, x_{n}\right\}$, a set of constraints $\mathcal{C}=\left\{c_{1}, \ldots, c_{m}\right\}$ and a set of domains $\left\{\left[x_{1}\right], \ldots,\left[x_{n}\right]\right\}$ containing the $x_{i}$ 's. Classically, the variables $x_{i}$ are real numbers or vectors, the constraints are equations between the variables (such as $\left.x_{3}=x_{1}+\exp \left(x_{2}\right)\right)$ and the domains are intervals [20], boxes [12], zonotopes [5] or ellipsoids [6]. The aim of propagation techniques is to contract as much as possible the domains for the variables without loosing any solution [4] [21]. With an interval approach, a random variable $x$ of $\mathbb{R}$ is often represented by an interval $[x]$ which encloses the support of its probability function. This representation is of course poorer than that provided by its probability density distribution, but it presents several advantages. (i) An interval representation is well adapted to represent random variables with imprecise probability density functions. (ii) An arithmetic can be developed for intervals [16], which makes it possible to deal with uncertainties in a reliable and easy way, even when strong nonlinearities occur. (iii) When the random variables are related by equations, contraction operators, called contractors [12], make it possible to get efficient polynomial algorithms to compute intervals that are guaranteed to contain all feasible values for the random variables. In this paper, we keep the CSP formalism, but the variables will be functions from $\mathbb{R}$ to $\mathbb{R}^{n}$ (or trajectories for short), the constraints will be equations on trajectories (such as differential equations, delay equations, etc.), and the domains will be tubes. Briefly, a tube is an interval of trajectories that can be represented in the computer and easily be handled. It is very similar to intervals or boxes, but tubes enclose trajectories instead of real numbers or vectors. The state estimation problem to be considered here can be represented by a CSP where the constraints are

$$
\left\{\begin{array}{l}
\dot{\mathbf{x}}(t)=\mathbf{f}(\mathbf{x}(t), t)+\mathbf{b}(t) \\
v(t)=h(\mathbf{x}(t)) \\
\dot{y}(t)=\frac{\partial g}{\partial \mathbf{x}}(\mathbf{x}(t)) * \dot{\mathbf{x}}(t) \\
y(t)=g(\mathbf{x}(t)) \\
v(t)=0 \Rightarrow y(t) \in \mathbb{W}(t) .
\end{array}\right.
$$

The variables of the CSP are the trajectories $\mathbf{x}(t), \dot{\mathbf{x}}(t)$, $\mathbf{b}(t), y(t), \dot{y}(t), v(t)$ and the domains are interval trajectories or tubes $[\mathbf{x}](t),[\dot{\mathbf{x}}](t),[\mathbf{b}](t),[y](t),[\dot{y}](t)$, $[v](t)$ containing the unknown trajectories. This CSP is equivalent to the initial state estimation problem (1), but a decomposition has been performed by introducing the trajectories $y(t), \dot{y}(t), v(t)$. Such a decomposition will allow us to deal with the constraints independently and will simplify the construction of the associated contractors.

The notion of tube we shall propose is similar to that classically used in the bounded-error community [13], [15]. The advantage of our definition is that it allows the use of interval arithmetic to compute with tubes. A tube $[\mathbf{x}](t)$, with a sampling time $\delta>0$, is a boxvalued function which is constant for all $t$ inside intervals $[k \delta, k \delta+\delta], k \in \mathbb{Z}$. The box $[k \delta, k \delta+\delta] \times[\mathbf{x}]\left(t_{k}\right)$, with $t_{k} \in[k \delta, k \delta+\delta]$ is called the $k$ th slice of the tube $[\mathbf{x}](t)$ and will be denoted by $[\mathbf{x}](k)$. A trajectory $\mathbf{x}(t)$ belongs to the tube $[\mathbf{x}](t)$ if $\forall t, \mathbf{x}(t) \in[\mathbf{x}](t)$. The notion of tube is illustrated by Figure 3 where the trajectory $v(t)$ is enclosed inside the tube $[v](t)$. This tube gives us the information related to the trajectory $v(t)$. For instance, from the tube we know that $v(t)$ has at least four roots (this is a consequence of the fact that we know the signs $v(t)$ inside the grey slices). If we additionally had a tube $[\dot{v}](t)$ for $\dot{v}(t)$ sufficiently tight, we could also conclude that $v(t)$ has exactly four roots inside the intervals $\left[t_{1}\right],\left[t_{2}\right],\left[t_{3}\right],\left[t_{4}\right]$.

We can extend some classical operations we have on trajectories (such as sums, multiplication, image by a function) to tubes. It suffices to perform the corresponding interval operations for all $t$ [16]. An arithmetic on tube is thus a direct extension of interval arithmetic. As it is the case for interval computation, the result of an operation on tubes contains all results of the same 


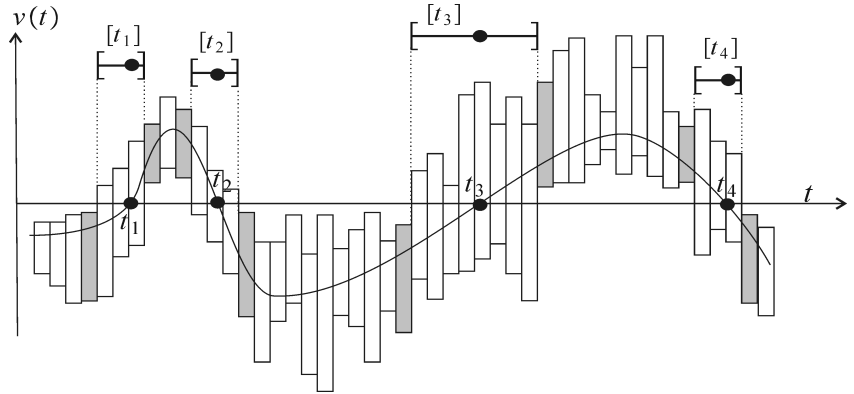

Fig. 3. A tube is a union of slices and encloses an uncertain trajectory

operation performed on the enclosed trajectories. Define the index correspondence function $\kappa$ as follows

$$
\kappa\left(\left[t_{a}, t_{b}\right]\right)=\left\{k \in \mathbb{Z}, \exists t \in\left[t_{a}, t_{b}\right], t \in[k \delta, k \delta+\delta]\right\} .
$$

The integral for tubes is defined by

$$
\int_{t_{0}}^{t}[\mathbf{x}](\tau) d \tau=\sum_{k \in \kappa\left(\left[t_{0}, t\right]\right)} \delta *[\mathbf{x}](k),
$$

where the addition rule for boxes $\delta *[\mathbf{x}](k)$ are provided by interval arithmetic. It is easy to prove that

$$
\mathbf{x}(t) \in[\mathbf{x}](t) \Rightarrow \int_{t_{0}}^{t} \mathbf{x}(\tau) d \tau \in \int_{t_{0}}^{t}[\mathbf{x}](\tau) d \tau
$$

and that the quantity $\int_{t_{0}}^{t}[\mathbf{x}](\tau) d \tau$ defines a tube. Except for atypical tubes, the derivative of a tube cannot be defined. However, in a state-estimation context, the state equations make possible to have an analytic expression of the derivatives. For instance, since $\dot{\mathbf{x}}(t)=$ $\mathbf{f}(\mathbf{x}(t), t)+\mathbf{b}(t)$ and $\dot{y}(t)=\frac{\partial g}{\partial \mathbf{x}}(\mathbf{x}(t)) * \dot{\mathbf{x}}(t)$, tubes enclosing the functions $\dot{\mathbf{x}}(t)$ and $\dot{y}(t)$ can be obtained by the following operations

$$
\begin{aligned}
& {[\dot{\mathbf{x}}](t):=[\mathbf{f}]([\mathbf{x}](t), t)+[\mathbf{b}](t)} \\
& {[\dot{y}](t):=\left[\frac{\partial g}{\partial \mathbf{x}}\right]([\mathbf{x}](t)) *[\dot{\mathbf{x}}](t)}
\end{aligned}
$$

where $[\mathbf{f}],\left[\frac{\partial g}{\partial \mathbf{x}}\right]$ are the interval counterparts of $\mathbf{f}, \frac{\partial g}{\partial \mathbf{x}}$. Tube arithmetic can be used to contract tubes with respect to algebraic constraints on trajectories. There exist also some techniques for the contraction of the tube $[\mathbf{x}](t)$ with respect to the differential constraint $\dot{\mathbf{x}}=\mathbf{f}(\mathbf{x}, t)$ [2], [3], [17].

\section{CONTRACTION OF THE VISIBILITY RELATION}

This section is devoted to the following problem. Given three tubes $[v](t),[y](t),[\dot{y}](t)$ associated with the trajectories $v(t), y(t), \dot{y}(t)$, contract the tubes $[v](t),[y](t)$ with respect to the relation

$$
v(t)=0 \Rightarrow y(t) \in \mathbb{W}(t) .
$$

We shall give two theorems. The first theorem will be used for the contraction of $[y](t)$ and the second theorem for the contraction of $[v](t)$.

Theorem 1. If $0 \in v([t])$ then for all $t$,

$$
y(t) \in \bigcup_{\tau \in[t]}\left((\mathbb{W}(\tau) \cap[y](\tau))+\int_{\tau}^{t}[\dot{y}](\alpha) d \alpha\right) .
$$

Proof. Assume that $0 \in v([t])$. Since $v(t)$ is a continuous function, from the Bolzano's theorem, $\exists \tau \in$ $[t], v(\tau)=0$. Now, from (4) and since $y(\tau) \in[y](\tau)$, we have

$$
y(\tau) \in \mathbb{W}(\tau) \cap[y](\tau) .
$$

Since $y(t)=y(\tau)+\int_{\tau}^{t} \dot{y}(\alpha) d \alpha$, from (6) and (3), we get $y(t) \in(\mathbb{W}(\tau) \cap[y](\tau))+\int_{\tau}^{t}[\dot{y}](\alpha) d \alpha$. Since $\tau \in[t]$, we get Equation (5).

A direct consequence of this theorem is that if $0 \in v([t])$ the tube $[y](t)$ for $y(t)$ can be contracted by intersecting it with the tube defined on the right hand side of Equation (5).

Corollary. If $0 \in v([t])$ and $0 \notin \dot{v}([t])$ then there exists a unique fleeting date in $[t]$.

This corollary can be used to count the number of detections, but is not used for tube contraction. This operation requires a tube for $\dot{v}(t)$ which can be obtained thanks to the relation $\dot{v}(t)=\frac{\partial v}{\partial \mathbf{x}}(\mathbf{x}(t)) * \dot{\mathbf{x}}(t)$. Note that an extension of Theorem 1 to the case where $v$ is a vector function can be obtained by using the mathematical tools presented in [9].

Theorem 2. We have the following implication

$$
\forall t \in[t],[y](t) \cap \mathbb{W}(t)=\emptyset \Rightarrow 0 \notin v([t]) .
$$

Proof. The proof is by contradiction. Assume that $0 \in$ $v([t])$ then, from the Bolzano's theorem, we have $\exists \tau \in$ $[t], v(\tau)=0$ and thus $y(\tau) \in \mathbb{W}(\tau)$. Since $y(\tau) \in$ $[y](\tau)$, we have $y(\tau) \in[y](\tau) \cap \mathbb{W}(\tau)$ which is in contradiction with the assumption.

A direct consequence of this theorem is that if for a given interval $[t]$ of $\mathbb{R}$ we have $\forall t \in[t],[y](t) \cap \mathbb{W}(t)=\emptyset$, the tube for $v(t)$ can be contracted by one of the following operations

$$
\begin{aligned}
& \exists t_{1} \in[t],[v]\left(t_{1}\right)>0, \text { then } \quad[v]([t]):=[v](t) \cap \mathbb{R}^{+} . \\
& \exists t_{2} \in[t],[v]\left(t_{2}\right)<0, \text { then } \quad[v]([t]):=[v]([t]) \cap \mathbb{R}^{-} .
\end{aligned}
$$

Note that the two conditions are mutually exclusive.

Example. Consider the following constraint satisfaction problem

$$
\left\{\begin{array}{c}
v(t)=0 \Rightarrow y(t) \in \mathbb{W}(t) \\
y(t)=y_{\tau}+\int_{\tau}^{t} \dot{y}(\alpha) d \alpha \\
v(t) \in[v](t), y(t) \in[y](t)
\end{array}\right.
$$




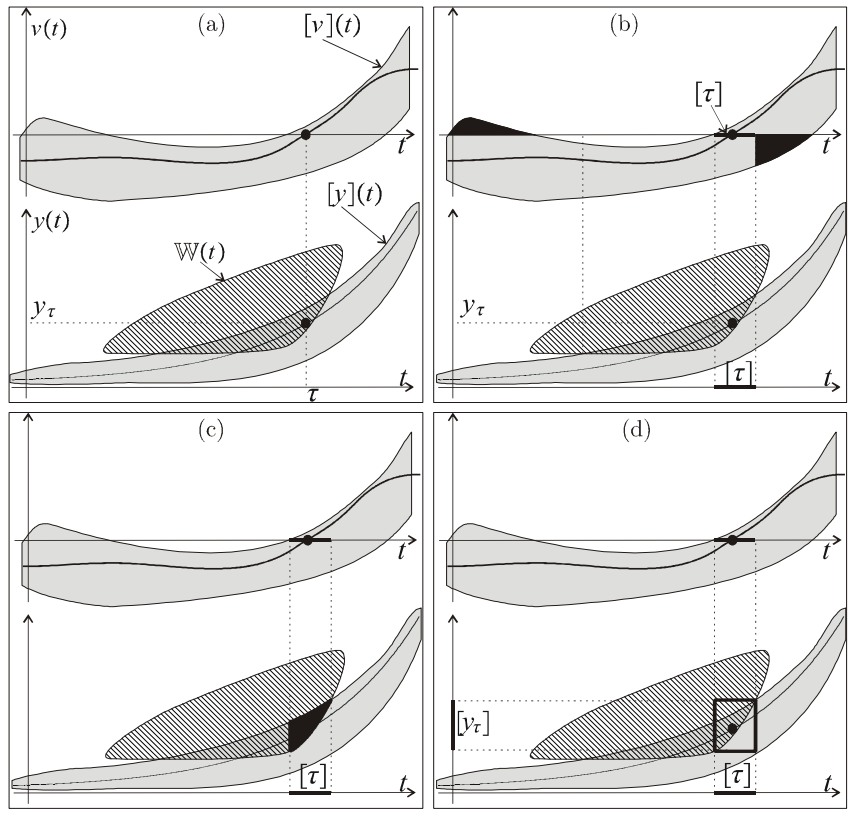

Fig. 4. Illustration of the contraction of tubes with respect to the visibility constraint

where $\mathbb{W}(t)$ and the initial tubes $[v](t),[y](t)$ are given by Figure 4 (a), below. Is also represented the unknown fleeting data point (small black point). Let us now show how $[v](t),[y](t)$ can be contracted. From Theorem 2, we are able to contract the tube $[v](t)$ by removing the black zones represented by Subfigure (b). We also deduce that there exists one $\tau \in[\tau]$ corresponding to a fleeting data point. Since this point should belong to both the waterfall $\mathbb{W}(t)$ and the tube $[y](t)$, it belongs to the black zone of Subfigure (c). The box $[\tau] \times\left[y_{\tau}\right]$ of subfigure (d) encloses a fleeting data point. We have

$$
y_{\tau} \in \bigcup_{\tau \in[\tau]}(\mathbb{W}(\tau) \cap[y](\tau)) .
$$

Equation (5) of Theorem 1 can then be used to contract the tube $[y](t)$.

\section{AlgORITHM}

As for all constraint propagation algorithms, we shall contract the domains for the variables until no more significant contraction can be observed. Recall that here the variables are trajectories, the domains are tubes and the constraints are given by (2).

Initialization. The first step is the initialization of tubes. All tubes are initialized with some prior conditions about the state. For instance, if some initial conditions are known for the state vector then the corresponding tube $[\mathbf{x}](t)$ will be contracted at time $t=0$. The waterfall $\mathbb{W}(t)$ is assumed to be known for all $t$. Thus, we consider here an offline state estimation problem.
Evolution contractor. Using differential interval techniques, we can get [2] accurate interval enclosures $\left[\varphi_{\delta}\right]$ for the flow $\varphi_{\delta}$ of the state equations. The contractor associated with the constraint $\dot{\mathbf{x}}(t)=\mathbf{f}(\mathbf{x}(t), t)+\mathbf{b}(t)$ will have the form

$$
\begin{aligned}
& \left.[\mathbf{x}](k+1):=[\mathbf{x}](k+1) \cap\left[\varphi_{\delta}\right]([\mathbf{x}](k), k \delta)\right) \\
& \left.[\mathbf{x}](k-1):=[\mathbf{x}](k-1) \cap\left[\varphi_{-\delta}\right]([\mathbf{x}](k), k \delta)\right) .
\end{aligned}
$$

These statements have to be performed for all $k$. A forward propagation followed by a backward propagation has been demonstrated [10] to be efficient in this context. There is no criteria able to forecast how accurate will be the enclosure. First, the set of all feasible state vectors can be arbitrarily large (because of the state noise or a large initial box). Moreover, interval methods always produce overestimation. This overestimation can be controlled using bisections or high order methods, but the computing time is increased. For treating our example, a simple first order interval method without bisection has been chosen.

Observation contractors. The contractions of the tubes $[\mathbf{x}](t),[\dot{\mathbf{x}}](t),[\dot{y}](t),[y](t),[v](t)$ with respect to the constraints $v(t)=h(\mathbf{x}(t)), \dot{y}(t)=\frac{\partial g}{\partial \mathbf{x}}(\mathbf{x}(t)) * \dot{\mathbf{x}}(t)$ and $y(t)=g(\mathbf{x}(t))$ can be performed [12] using some classical interval constraint propagation.

Visibility contractors. The relation $v(t)=0 \Rightarrow y(t) \in$ $\mathbb{W}(t)$ can be used to contract the tubes $[v](t)$ and $[y](t)$. The contraction of the tube $[y](t)$ is based on Theorem 1 and is illustrated by Figure 5. Let us now describe the method. 1) We take two slices $[v]\left(k_{1}\right)$ and $[v]\left(k_{2}\right)$ such that $0 \in[v](k)$ for all $k \in\left[k_{1}+1, k_{2}-1\right]$ and such that $[v]\left(k_{1}\right)$ and $[v]\left(k_{2}\right)$ have opposite signs (see subfigure (a)). 2) For each $k \in\left[k_{1}+1, k_{2}-1\right]$, we compute the subtube $(\mathbb{W}(\tau) \cap[y](\tau))+\int_{\tau}^{t}[\dot{y}](\alpha) d \alpha$ (see ,subfigures (b) and (c)). 3) We compute the union of the resulting subtubes and intersect this union with the initial tube $[y](t)$ (see subfigure $(d)$ ).

The contraction of the tube $[v](t)$ is based on Theorem 2 . The principle is to find a subtube $\left\{[y]\left(k_{1}\right), \ldots,[y]\left(k_{2}\right)\right\}$ that does not intersect $\mathbb{W}(t)$. Since all corresponding trajectories $v(t)$ should have the same sign, the subtube $\left\{[v]\left(k_{1}\right), \ldots,[v]\left(k_{2}\right)\right\}$ can be contracted.

\section{TEST-CASE}

Consider a robot moving on a plane and equipped with a directive laser rotating rangefinder (see [14] and [19] for more about this type of problem). We shall assume that the dynamic of the robot is described by the following 

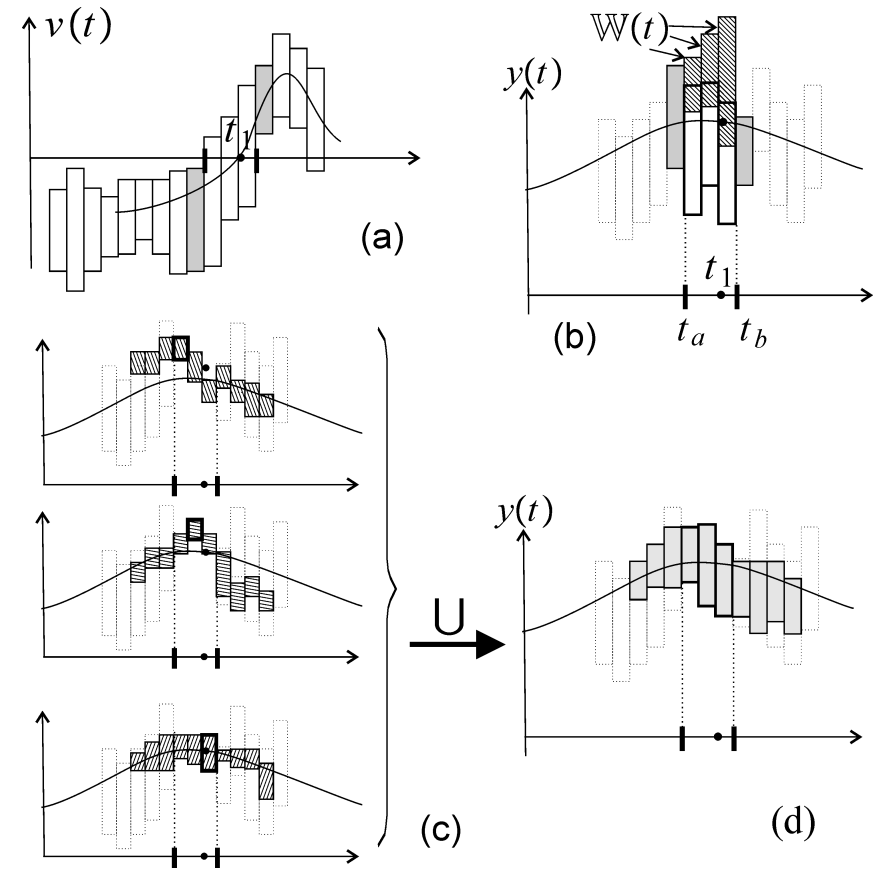

(c)

(d)

Fig. 5. Contraction of the tube $[y]$ when we know (from the sign of $v$ ) that a visible data has been detected

state equations

$$
\left\{\begin{array}{l}
\dot{x}_{1}=\cos x_{3}+b_{1} \\
\dot{x}_{2}=\sin x_{3}+b_{2} \\
\dot{x}_{3}=u+b_{3} \\
\dot{x}_{4}=\omega+b_{4} .
\end{array}\right.
$$

where, $u(t)$ corresponds to the control. The pair $\left(x_{1}, x_{2}\right)$ corresponds to the coordinates of the robot, $x_{3}$ is its orientation and $x_{4}$ is the laser angle (which rotates at a speed of $\left.\omega=2 \mathrm{rad} . \mathrm{sec}^{-1}\right)$. Figure 6 provides a representation of the robot. The triangle and the square are detected by the laser but their presence is initially ignored by the robot. We shall assume that both $x_{3}$ and $x_{4}$ are measured with a given accuracy of \pm 0.01 . The initial condition is known to belong to the box $[-2 \mathrm{~m}, 2 \mathrm{~m}] \times[-7 \mathrm{~m},-3 \mathrm{~m}] \times[-2 \mathrm{rad}, 2 \mathrm{rad}] \times[-2 \mathrm{rad}, 2 \mathrm{rad}]$. The state noises $b_{i}(t)$ are uniformly distributed inside the interval $[-1 \mathrm{~cm}, 1 \mathrm{~cm}]$. A mark $\mathbf{m}$ is located at coordinates $(0 \mathrm{~m}, 0 \mathrm{~m})$ and the distance between the robot and the mark is measured with an accuracy of $\pm 1 \mathrm{~cm}$ only if the mark is exactly pointed by the laser beam and if no obstacle lies between the robot and the mark. The scope of the rangefinder corresponds to the interval $[s]=\left[s^{-}, s^{+}\right]=[1 \mathrm{~m}, 10 \mathrm{~m}]$. Define the visibility and the observation functions as

$$
\left\{\begin{array}{l}
h(\mathbf{x})=x_{1} \sin \left(x_{3}+x_{4}\right)-x_{2} \cos \left(x_{3}+x_{4}\right) \\
g(\mathbf{x})=-x_{1} \cos \left(x_{3}+x_{4}\right)-x_{2} \sin \left(x_{3}+x_{4}\right) .
\end{array}\right.
$$

Denote by $d(t)$ the distance of the robot to the first obstacle following the direction pointed by the laser. If

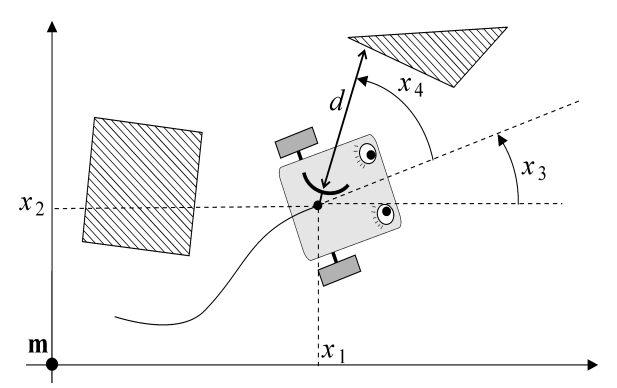

Fig. 6. The robot equipped with a rotating telemeter has to localize itself. It measures the distance $d$ to the first obstacle. Only the location of the mark $\mathbf{m}$ is known.

the first obstacle is inside the scope of the rangefinder, it returns an interval distance $[d]=\left[d^{-}, d^{+}\right]$which contains $d$. The mark $\mathbf{m}$ is seen by the laser if $(i)$ the line corresponding to the laser beam contains $\mathbf{m}$ (i.e., $h(\mathbf{x})=0$ ), (ii) the distance between the robot and the mark is inside the range of the rangefinder $\left(g(\mathbf{x}) \in[s]=\left[s^{-}, s^{+}\right]\right)$and (iii) there is no object between the robot and the mark $(\neg(d<g(\mathbf{x})))$. We thus have the relation

$$
(h(\mathbf{x})=0 \wedge g(\mathbf{x}) \in[s] \cap[-\infty, d]) \Rightarrow d=g(\mathbf{x}) .
$$

Now, from the following logical equivalences $(A \wedge B \Rightarrow C) \Leftrightarrow(A \Rightarrow \neg B \vee C)$, this implication translates into

$$
h(\mathbf{x})=0 \Rightarrow(g(\mathbf{x}) \notin([s] \cap[-\infty, d]) \vee d=g(\mathbf{x})) .
$$

or equivalently

$$
h(\mathbf{x})=0 \Rightarrow g(\mathbf{x}(t)) \in\left[-\infty, s^{-}\right] \cup\left[s^{+}, \infty\right] \cup[d, \infty] .
$$

Since $d^{-} \leq d$, we get the fleeting state estimation problem (1), with

$$
\mathbb{W}(t)=\left[-\infty, s^{-}\right] \cup\left[s^{+}, \infty\right] \cup\left[d^{-}, \infty\right] .
$$

Generation of the data. Let us take a constant control $u(t)=0.2 \mathrm{rad} . \mathrm{sec}^{-1}$, an initial state $\mathbf{x}_{0}=$ $(0 \mathrm{~m},-5 \mathrm{~m}, 0 \mathrm{rad}, 0 \mathrm{rad})^{\mathrm{T}}$, a sampling time $\delta=0.02$ $\mathrm{sec}$ and $t \in[0 \mathrm{sec}, 40 \mathrm{sec}]$. We have chosen a room which is almost a square and two small obstacles moving inside the room. The mark $\mathbf{m}$ is represented by the small black square at the top of the triangle at the centre of the room. An illustration of the mission performed by the robot is depicted on Figure 7 for $t \in\{0 \mathrm{sec}, 5 \mathrm{sec}, \ldots, 35 \mathrm{sec}\}$. For $t=35 \mathrm{sec}$, the first obstacle is beyond the scope of the rangefinder.

Figure 8 represents $\mathbb{W}(t)$ inside the frame $t \in$ $[0 \mathrm{sec}, 40 \mathrm{sec}], d \in[1 \mathrm{~m}, 10 \mathrm{~m}]$. The circles correspond to the fleeting data point $(t, g(\mathbf{x}(t)))$. All of them belong to $\mathbb{W}(t)$. When a circle corresponds to a point that is 


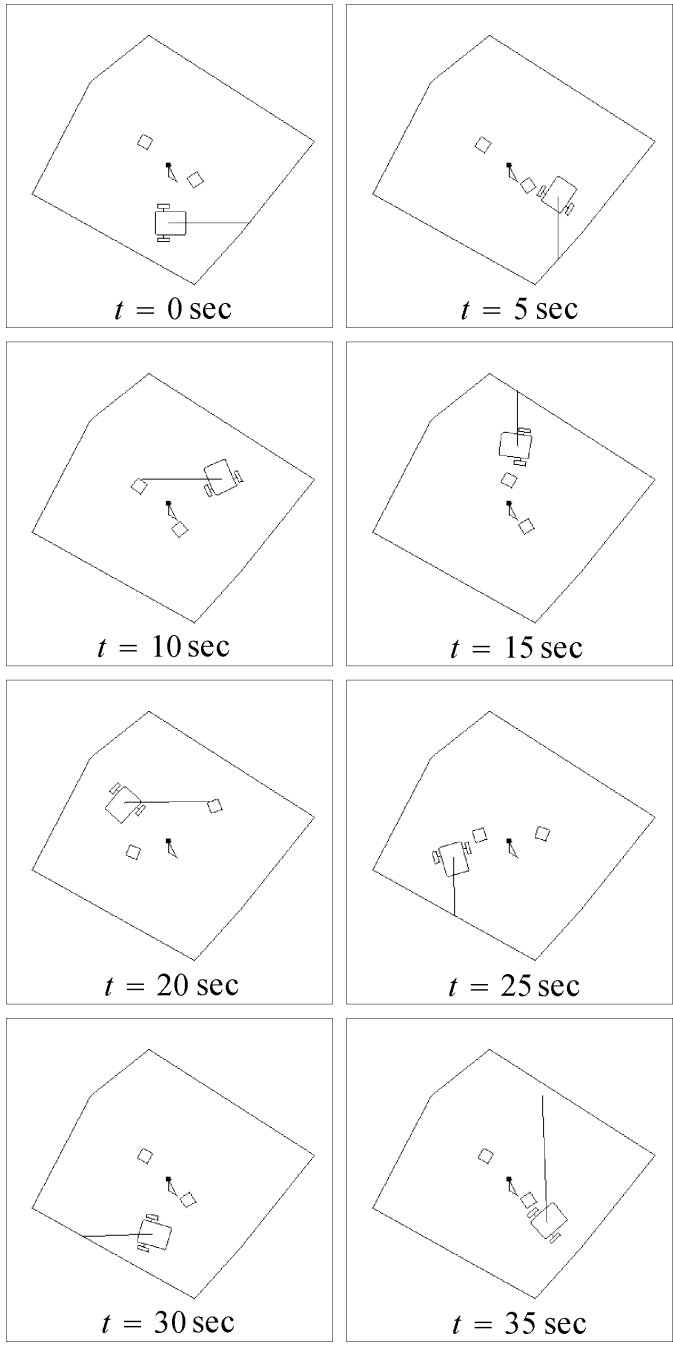

Fig. 7. Robot for different $t$ during the mission

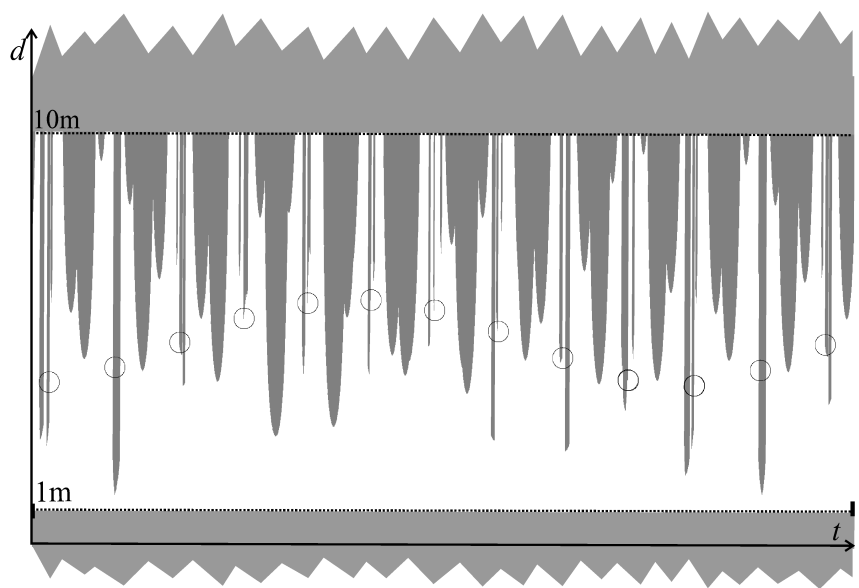

Fig. 8. The robot only knows that the fleeting data points $(t, g(\mathbf{x}(t))$ always lie inside the waterfall $\mathbb{W}(t)$ (painted grey) or equivalently that no fleeting data points are inside the white area
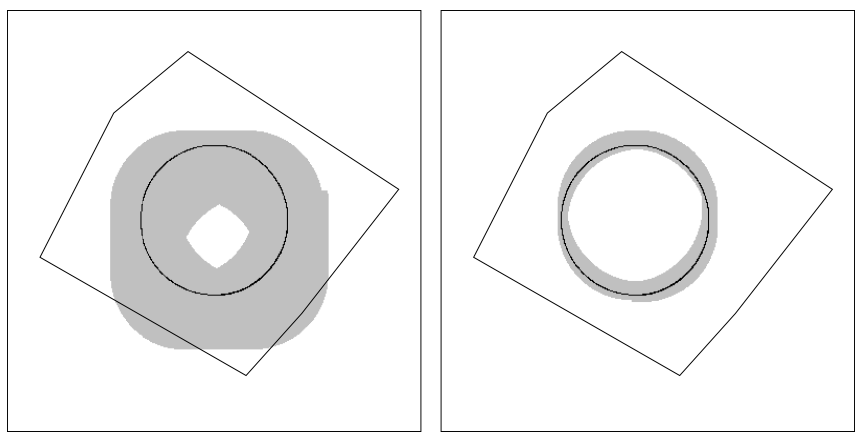

Fig. 9. Left: envelope obtained by an interval integration without using the rangefinder. Right: the information collected by the rangefinder is taken into account

on the bottom boundary of $\mathbb{W}$, it means that the mark is detected by the laser.

Envelope of the trajectory. Recall that the robot does neither know the map nor the existence of the moving obstacles. The only thing it knows is the location of the mark $\mathbf{m}$. When the laser rangefinder returns an interval distance in the interval $[d]=[4.99 \mathrm{~m}, 5.01 \mathrm{~m}]$, the robot translates this information into "the mark $\mathbf{m}$ is not in part of the beam segment which is at a distance of the robot inside $[1 \mathrm{~m}, 4.99 \mathrm{~m}] "$. In fact, the robot measures the absence of mark inside a part of the beam segment rather than its distance to the mark. Figure 9, left provides an interval envelope (painted grey) of the trajectory after a simple interval propagation which does not take into account the data collected by the rangefinder. The length of the initial box is $4 \mathrm{~m}$ and $6 \mathrm{~m}$ for the final box. After an interval propagation which takes into account the rangefinder, we get the trajectory of Figure 9 (right) and the precision of the localization is less than one meter for all $t$. The frame boxes are $[-14 \mathrm{~m}, 14 \mathrm{~m}]^{2}$ for both subfigures. The actual trajectory corresponds to the black circle.

Constructing the map. Denote by $[\mathbf{x}](t)$ the boxes obtained after the completion of the interval propagation and by $d(t)$ the distance returned by the rangefinder. An outer approximation of the set

$$
\mathbb{M}=\left\{\begin{array}{l}
\left(z_{1}, z_{2}\right), \exists t \in[0 \mathrm{sec}, 40 \mathrm{sec}], \exists \mathbf{x} \in[\mathbf{x}](t) \\
z_{1}=x_{1}(t)+d(t) * \cos \left(x_{3}(t)+x_{4}(t)\right), \\
\left.z_{2}=x_{2}(t)+d(t) * \sin \left(x_{3}(t)+x_{4}(t)\right)\right\}
\end{array}\right.
$$

can easily be computed by a simple interval evaluation. We obtain the set of boxes depicted on Figure 10, left. This picture gives an outer approximation of the map of the surrounding environment of the robot. Figure 10 right represents the center of all boxes covering $\mathbb{M}$.

Some movies illustrating the simulation and the resolution, as well as the $\mathrm{C}++$ source code of the programs 


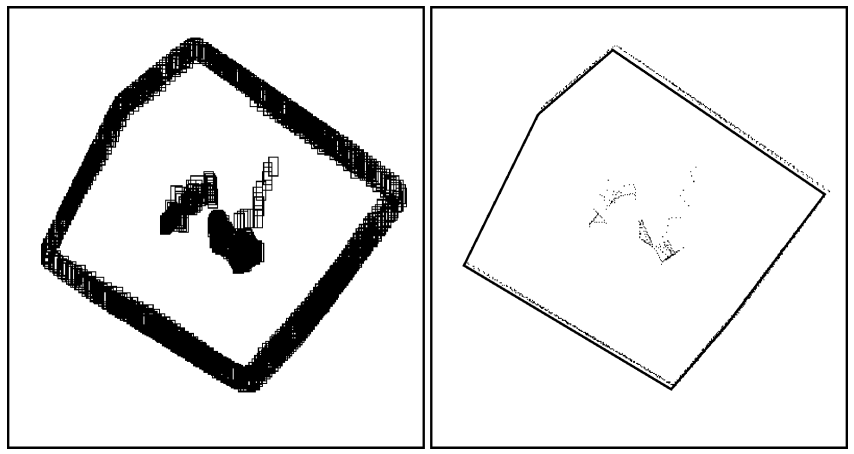

Fig. 10. Left: set of boxes, the union of which encloses the map; Right: approximation of the map made with the center of the previous boxes

can be downloaded at

WwW.ensta-bretagne.fr/lebars/fleeting/

\section{CONCLUSION}

In this paper, an interval approach has been proposed to deal with offline state estimation in the case where fleeting data are involved. To our knowledge, existing approaches cannot be used to deal with such estimation problems. The difficulty of the problem comes from the nature of the data that are significant only for some given fleeting dates $t$ that are unknown. The problem is transformed into a constraint satisfaction problem where the variables are trajectories and the domains are tubes. This transformation made possible to use a constraint propagation approach. The resulting method has been illustrated on the dynamic localization of a wheeled robot in an encumbered moving environment where the location of a single mark is known. For this problem, the single exteroceptive sensor that is used is a rotating laser rangefinder. The localization is then used to reconstruct the map of the surrounding environment.

\section{REFERENCES}

[1] F. Abdallah, A. Gning, and P. Bonnifait. Box particle filtering for nonlinear state estimation using interval analysis. Automatica 44(3), 807-815 (2008).

[2] M. Berz, C. Bischof, G. Corliss, And G. A., editors. "Computational Differentiation: Techniques, Applications and Tools". SIAM, Philadelphia, Penn. (1996).

[3] M. BERZ AND K. MAKINO. Verified integration of odes and flows using differential algebraic methods on high-order taylor models. Reliable Computing 4(3), 361-369 (1998).

[4] G. Chabert and L. Jaulin. Contractor Programming. Artificial Intelligence 173, 1079-1100 (2009).

[5] C. Combastel. A state bounding observer for uncertain nonlinear continuous-time systems based on zonotopes. In "CDCECC '05' (2005).
[6] C. Durieu, B. Polyak, And E. Walter. Ellipsoidal state outer-bounding for MIMO systems via analytical techniques. In "Proceedings of the IMACS-IEEE-SMC CESA'96 Symposium on Modelling and Simulation", vol. 2, pp. 843-848, Lille, France (1996).

[7] J. GLYNN. "Acoustic calibration and bathymetric processing with a KLEIN 5410 sidescan sonar". PhD thesis, University of New Hampshire, US (2007).

[8] A. GNing AND P. Bonnifait. Constraints propagation techniques on intervals for a guaranteed localization using redundant data. Automatica 42(7), 1167-1175 (2006).

[9] A. GoldSZTEJN AND L. JAULIN. Inner and outer approximations of existentially quantified equality constraints. In "Proceedings of the Twelfth International Conference on Principles and Practice of Constraint Programming, (CP 2006)", Nantes (France) (2006).

[10] L. JAULIN. Nonlinear bounded-error state estimation of continuous-time systems. Automatica 38, 1079-1082 (2002).

[11] L. JAulin, M. KiefFer, I. Braems, AND E. WAlter. Guaranteed nonlinear estimation using constraint propagation on sets. International Journal of Control 74(18), 1772-1782 (2001).

[12] L. Jaulin, M. Kieffer, O. Didrit, And E. Walter. "Applied Interval Analysis, with Examples in Parameter and State Estimation, Robust Control and Robotics". Springer-Verlag, London (2001)

[13] A. Kurzhanski And I. VAlyi. "Ellipsoidal Calculus for Estimation and Control". Birkhäuser, Boston, MA (1997).

[14] J. Leonard And H. DurRant-Whyte. Dynamic Map Building for an Autonomous Mobile Robot. International Journal of Robotics Research 11(4) (1992).

[15] M. Milanese, J. Norton, H. Piet-Lahanier, And E. WALTER, editors. "Bounding Approaches to System Identification”. Plenum Press, New York, NY (1996).

[16] R. E. MoOre. "Methods and Applications of Interval Analysis". SIAM, Philadelphia, PA (1979).

[17] T. Raissi, N. Ramdani, AND Y. Candau. Set membership state and parameter estimation for systems described by nonlinear differential equations. Automatica 40, 1771-1777 (2004).

[18] D. SAM-HAROUD. "Constraint consistency techniques for continuous domains". PhD dissertation 1423, Swiss Federal Institute of Technology in Lausanne, Switzerland (1995).

[19] S. Thrun, D. Fox, W. Burgard, AND F. Dellaert. Robust Monte Carlo localization for mobile robots. Artificial Intelligence 128, 99-141 (2000).

[20] M. VAN EMDEN. Algorithmic power from declarative use of redundant constraints. Constraints 4(4), 363-381 (1999).

[21] P. VAn Hentenryck, L. Michel, And Y. Deville. "Numerica - A Modelling Language for Global Optimization". MIT Press, Cambridge, Massachusetts (1997).

[22] G. VideAu, T. RAÏssi, AND A. Zolghadri. Guaranteed state estimation for nonlinear continuous-time systems based on qlpv transformations. In "Proceedings of European Control Conference (ECCŠ09)", Budapest, Hungary (2009).

[23] E. Walter and L. Pronzato. "Identification of Parametric Models from Experimental Data”. Springer-Verlag, London, UK (1997). 